\title{
Endoscopic Management of Benign Tumors of Paranasal Sinus and Nasal Cavity: An Institutional Experience
}

\author{
Rajat Jain, M. Ravi Sankar, Surendra Singh Bhagel, Pearly P. K., Neha Singh, Amit Keshri* \\ Neuro-otology Unit, Department of Neurosurgery, Sanjay Gandhi Institute of Medical Sciences, Lucknow, U.P, India.
}

How to cite this paper: Rajat Jain, M. Ravi Sankar, Surendra Singh Bhagel, Pearly P. K., Neha Singh, Amit Keshri. (2021) Endoscopic Management of Benign Tumors of Paranasal Sinus and Nasal Cavity: An Institutional Experience. International Journal of Clinical and Experimental Medicine Research, 5(2), 139-148.

DOI: $10.26855 /$ ijcemr.2021.04.008

Received: January 31, 2021

Accepted: February 28, 2021

Published: March 30, 2021

*Corresponding author: Amit Keshri, Neuro-otology Unit, Department of Neurosurgery, Sanjay Gandhi Institute of Medical Sciences, Lucknow, U.P, India.

Email: drkeshriamit@gmail.com

\begin{abstract}
Paranasal sinus (PNS) and nasal cavity tumors are rare, and account for $3 \%$ of all head and neck malignancies. Experience with endoscopic technique, advances in visualization, introduction of image guidance and development of surgical tools has led to a shift towards the treatment of PNS and nasal tumors through endoscopic approaches. Retrospectively study was done in a tertiary care institute from January 2008 to December 2018. Inclusion criteria—males and females irrespective of age, histologically proven benign tumors of paranasal sinus and nasal cavity. Exclusion criteria-patients diagnosed with juvenile nasoangiofibroma (JNA), histologically proven malignant tumors of paranasal and nasal cavity, involvement of skin, orbital extension, intracranial extension of tumor. 26 patients of benign paranasal sinus malignancy were operated, male-14, female-12. Most common being inverted papilloma (n-16, 61.5\%), Benign PNS and nasal cavity tumors not involving skin, orbit, tumors with intracranial extension can be managed by endoscopic excision.
\end{abstract}

\section{Keywords}

Endoscopic, Benign, PNS, Management, Tumor

\section{Introduction}

Paranasal sinus (PNS) and nasal cavity tumors are rare, and account for 3\% of all head and neck malignancies [1-3]. Due to nonspecific presenting symptoms, they are diagnosed at an advanced stage. Tumors of PNS and nasal cavity are divided into benign and malignant group. Factors such as histopathological type, site of origin, growth pattern, involvement of neighboring structures influence their prognosis $[4,5]$. The presenting features and symptomatology and advanced imaging technique help to reach a presumptive diagnosis, but histopathological examination remains the mainstay of definitive diagnosis. Endoscopic sinus surgery was initially introduced for the treatment of chronic rhinosinusitis and limited benign tumors of paranasal sinuses. Currently this approach is considered for the treatment of majority of benign and malignant tumors of paranasal sinus and nasal cavity [6]. Experience with endoscopic technique, advances in visualization, introduction of image guidance and development of surgical tools has led to a shift towards the treatment of PNS and nasal tumors through endoscopic approaches. The aim of this clinic opathological study is to present endoscopic management of benignnasal and paranasal sinus tumors.

\subsection{Aim}

The aim of this study is to present the endoscopic management of benigntumors of paranasal sinus and nasal cavity. 


\section{Material and methods}

Retrospectively study was done in a tertiary care institute from January 2008 to December 2018. Inclusion criteria-males and females irrespective of age, histologically proven benign tumors of paranasal sinus and nasal cavity. Exclusion criteria — patients diagnosed with juvenile nasoangiofibroma (JNA), histologically proven malignant tumors of paranasal and nasal cavity, involvement of skin, orbital extension, intracranial extension of tumor.

\subsection{Preoperative settings}

Diagnostic nasal endoscopy with trans nasalbiopsy (excluding JNA), CECT PNS axial and coronal cuts $1 \mathrm{~mm}$ thick along with MRI PNS with contrast.

\subsection{Post operative setting}

CECT PNS axial and coronal cuts $1 \mathrm{~mm}$ thick were done after 4 weeks of surgery followed by radiotherapy if needed. The data on tumor type, operative technique, perioperative complications and postoperative course were analyzed.

\subsection{Observations}

26 patients of benignparanasal sinus malignancy were operated, male-14, female-12. Most common being inverted papilloma (n-16, 61.5\%), plasma cell proliferative disorder (n-2, 7.69\%), glomangiopericytoma (n-1, 3.8\%), fibrous dysplasia (n-1,3.8\%), giant cell reparative granuloma (n-1,3.8\%), myxofibroma (n-1,3.8\%), hemangiopericytoma (n-1, 3.8\%), schwannoma (n-1, 3.8\%), amyloidosis (n-1, 3.8\%), ossifying fibroma(n-1, 3.8\%) (Figure 6). Post-operativeradiotherapy was given in one case of inverted papilloma showing carcinoma in situ on histopathological examination and one case of plasma cell proliferative disorder. The mean observation period was 17.3 months (range: 4-60 months).

\subsection{Operative technique and post-operative care}

Procedures performed under general anesthesia. Nasal mucosa decongested using adrenaline-soaked cotton patties, nasal cavity was inspected with a $0^{\circ}$ endoscope. Ethmoidectomy, septectomy was done to gain access around tumor and identify site of attachment. If needed tumor was debulked using power instruments like debrider or coblation. Once origin of the tumor was identified it was removed with a margin and the underlying bone was removed. Extension of the procedure depended on specific pathology, tumor advancement and individual anatomic variations. Saline nasal douches were advised for 6 weeks after the surgery. Follow-up visits with debridement of crusts under endoscopic view was carried out every 30 days for at least 3 months, and then at least once in 3 months for 2 years and then yearly up to 5 years. Follow-up imaging was performed using computed tomography 4 weeks following surgery to see any residual or recurrence.

\section{Discussion}

Main argument against endoscopic surgery of Sino nasal and skull-base tumors is piecemeal resection rather than en bloc resection, which is easier to achieve with open approaches. However, the concept of oncology remains same, i.e. R0 resection. In our study, we have tried to achieve tumor free margins in all cases with resection of one more layer endoscopically. If tumor was found involving critical structures like carotid, cavernous sinus it was left at that place and radiotherapy was given to prevent its growth in that region. Proponents of endoscopic surgery underline its minimal invasiveness and low morbidity [7]. Several studies have suggested that in selected cases endoscopic approaches are as efficient as open ones [8,9]. Open approaches can be assisted endoscopically to have better visualization in deeper area, like close to carotid, cavernous sinus, dura, orbit. Endoscopic assistance in these areas promotes better dissection. We will be discussing few benign paranasal sinus and nasal cavity malignancies in our study and we have tried to give a management protocol for the same. V J Lund et al. [32] have given United Kingdom national multidisciplinary guidelines for nose and paranasal sinus tumour. We fully advocate these guidelines and strongly recommend them to be followed for all benign pns tumours.

3.1 Inverted papilloma/Squamous papilloma/Ringertz tumor (n-16, 61.5\%) was the most common benign tumor in our study. It comprises about $0.5 \%-4 \%$ of all primary nasal tumor. Clinically, it seems to be arising from lateral nasal wall (Figure 1), with involvement of at least one paranasal sinus having male predominance in fifth and sixth decade of life [34]. Common symptoms include unilateral nasal obstruction, epistaxis and sinusitis with nasal discharge. Although the intent of surgical procedures was curative, recurrence rates of $40 \%-80 \%$ were unac- 
ceptably high earlier as people used to do only polypectomies. "Recurrence" represents residual disease in most cases. Up to 3\%-4\% of Inverted papillomas tend to convert into squamous cell carcinoma [10]. Open approaches such as the lateral rhinotomy and midfacial degloving allowed for increased tumor visualization and more complete resection including maxillectomy, which minimizes the recurrence rates. While with the advent of endoscopic surgery, inverted papilloma can be very well resected using a modified denkers procedure. Krause staging is the most accepted staging for inverted papilloma [11]. In our study, we did excision of the tumor via transnasal endoscopic modified denkers approach and achieved full piecemeal removal followed by drilling of bone in involved area (Figure 1). No recurrence was identified in any with a follow up of 5 years. One patient histopathology showed inverted papilloma with carcinoma in situ. Post-surgery adjuvant therapy in the form of radiotherapy was given to patient, five year follow up showed no recurrence (Table 1) (Figure 7).

Table 1. Distribution of benign PNS tumour, peri-operative complications, Post-operative complications/Radiotherapy/Recurrence

\begin{tabular}{|c|c|c|c|c|c|c|c|}
\hline S.no & Tumor subgroup & $\begin{array}{l}\text { Number of } \\
\text { patients (n) }\end{array}$ & $\begin{array}{l}\text { Male /female } \\
\text { ratio }\end{array}$ & $\begin{array}{l}\text { Perioperative } \\
\text { complication }\end{array}$ & $\begin{array}{l}\text { Post-operative } \\
\text { complication }\end{array}$ & Radiotherapy & Recurrence \\
\hline 1. & Inverted papilloma & $16(61.5 \%)$ & $m-8, f-8$ & none & - & $\begin{array}{c}\text { Yes in one } \\
\text { patient }\end{array}$ & no \\
\hline 2. & $\begin{array}{c}\text { Plasma cell proliferative } \\
\text { disorder }\end{array}$ & $2(7.7 \%)$ & $\mathrm{m}-1, \mathrm{f}-1$ & none & - & $\begin{array}{l}\text { Yes in one } \\
\text { patient }\end{array}$ & no \\
\hline 3. & Glomangiopericytoma & $1(3.8 \%)$ & $\mathrm{m}-1$ & none & - & - & no \\
\hline 4. & Fibrous dysplasia & $1(3.8 \%)$ & $\mathrm{m}-1$ & none & - & - & no \\
\hline 5. & $\begin{array}{l}\text { Giant cell reparative } \\
\text { granuloma }\end{array}$ & $1(3.8 \%)$ & $\mathrm{m}-1$ & none & - & - & no \\
\hline 6. & Myxofibroma & $1(3.8 \%)$ & $\mathrm{m}-1$ & none & - & - & no \\
\hline 7. & Schwannoma & $1(3.8 \%)$ & $\mathrm{f}-1$ & none & - & - & no \\
\hline 8. & Amyloidosis & $1(3.8 \%)$ & $\mathrm{m}-1$ & none & - & - & no \\
\hline 9. & Ossifying fibroma & $1(3.8 \%)$ & $f-1$ & none & - & - & no \\
\hline 10. & Hemangiopericytoma & $1(3.8 \%)$ & $\mathrm{f}-1$ & none & - & - & no \\
\hline
\end{tabular}

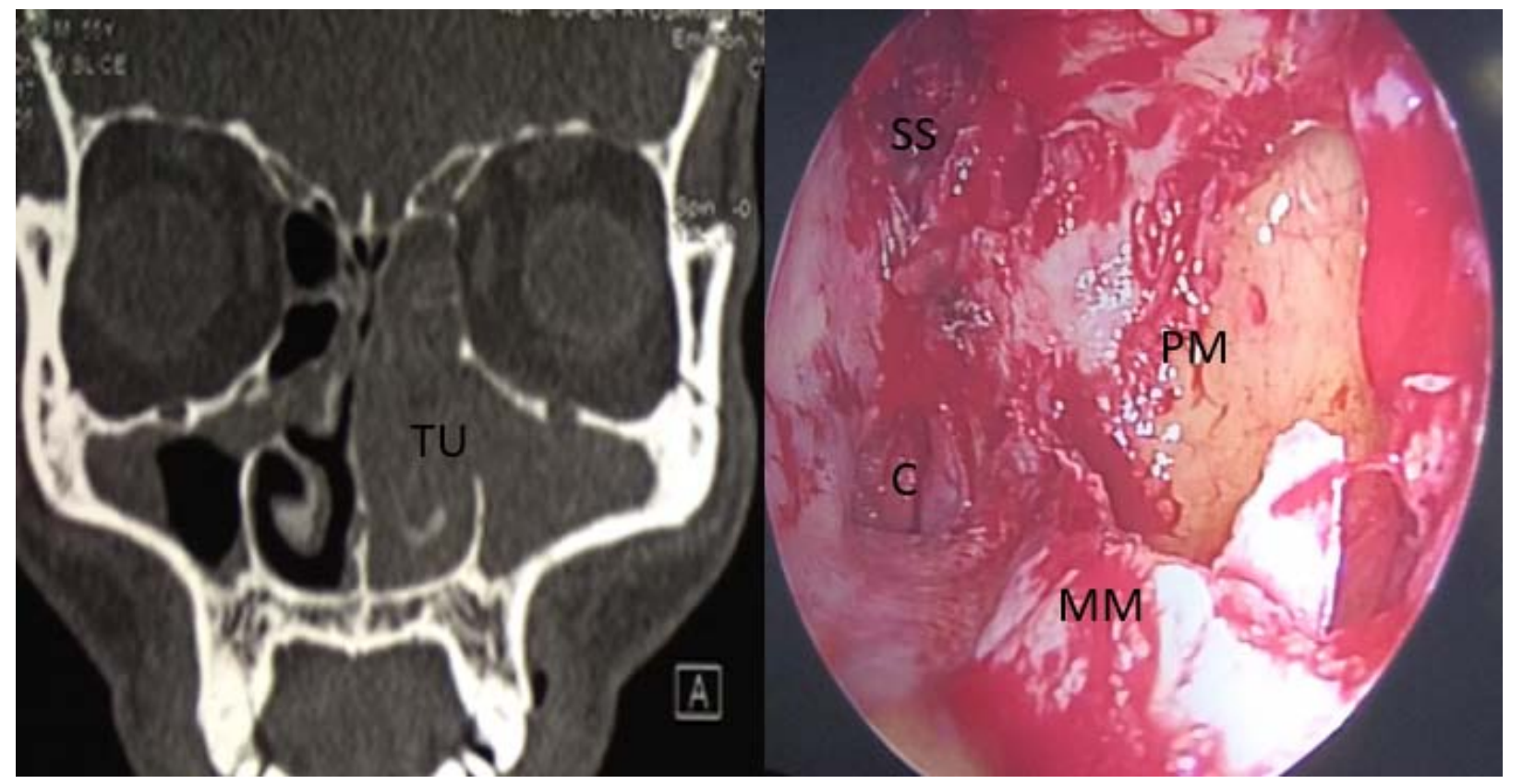

Figure 1. Right sided CT image showing tumor (TU) in left maxillary sinus and ethmoid region, left sided intraoperative picture shows total removal of tumor. SS-sphenoid sinus, C-choanae, PM-Posterior wall of maxilla, MM-Medial wall of maxilla. 


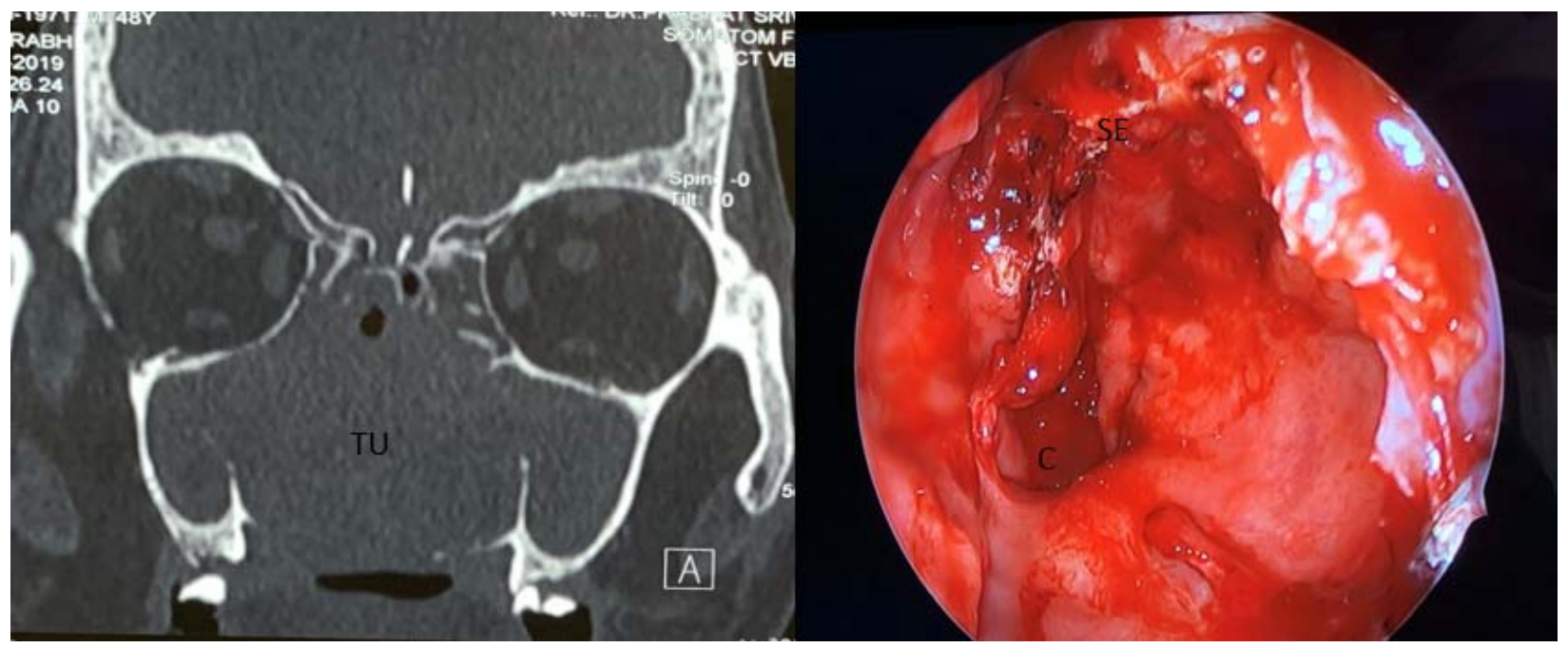

Figure 2. Right sided CT image showing tumour in both maxillary sinus, ethmoid sinus with erosion of septum. Left sided Intraoperative endoscopic image shows total removal of tumour. C-Choanae, SE-Remnant of septum.

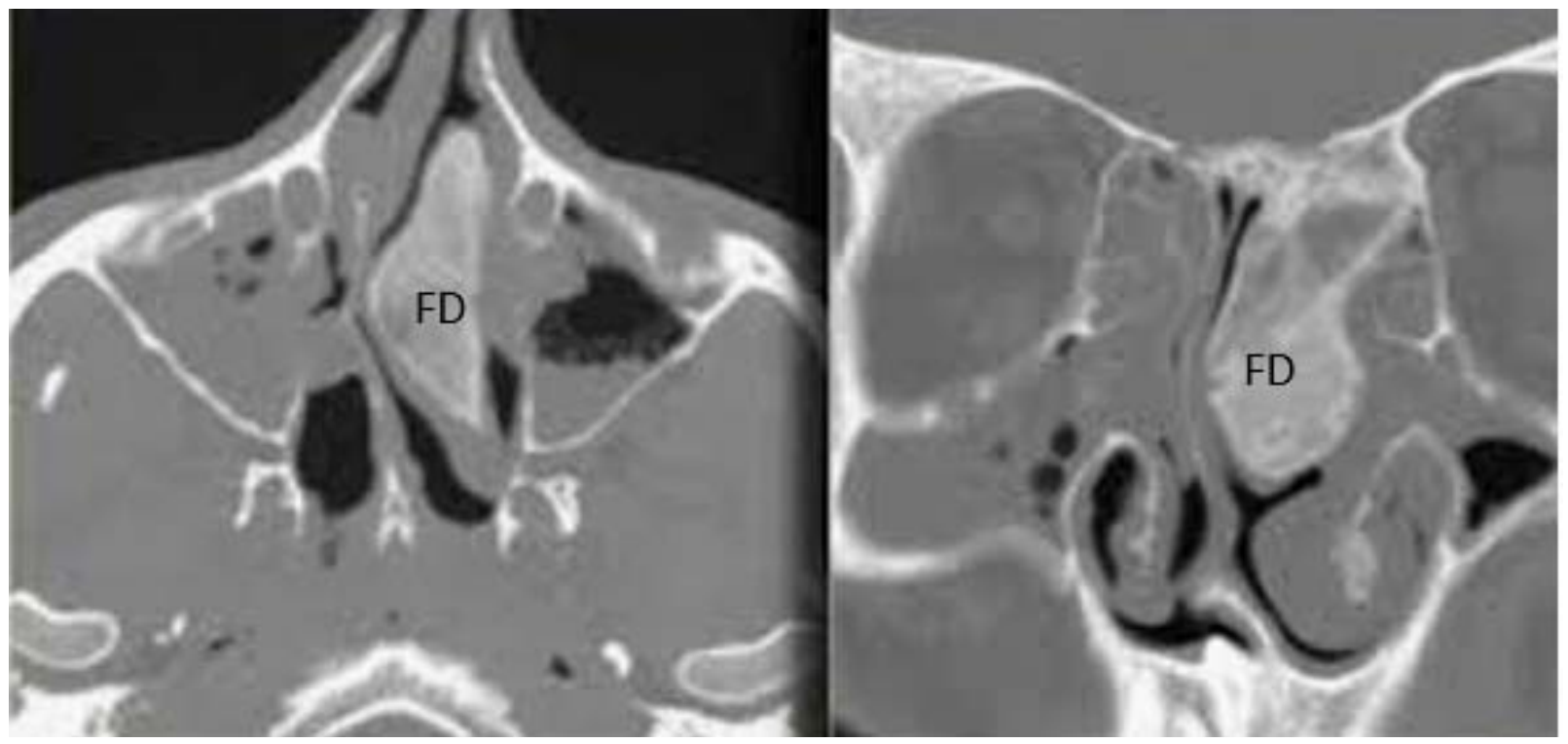

Figure 3. CT PNS axial and coronal sections showing ground glass appearance in ethmoidal region. FD-fibrous dysplasia.

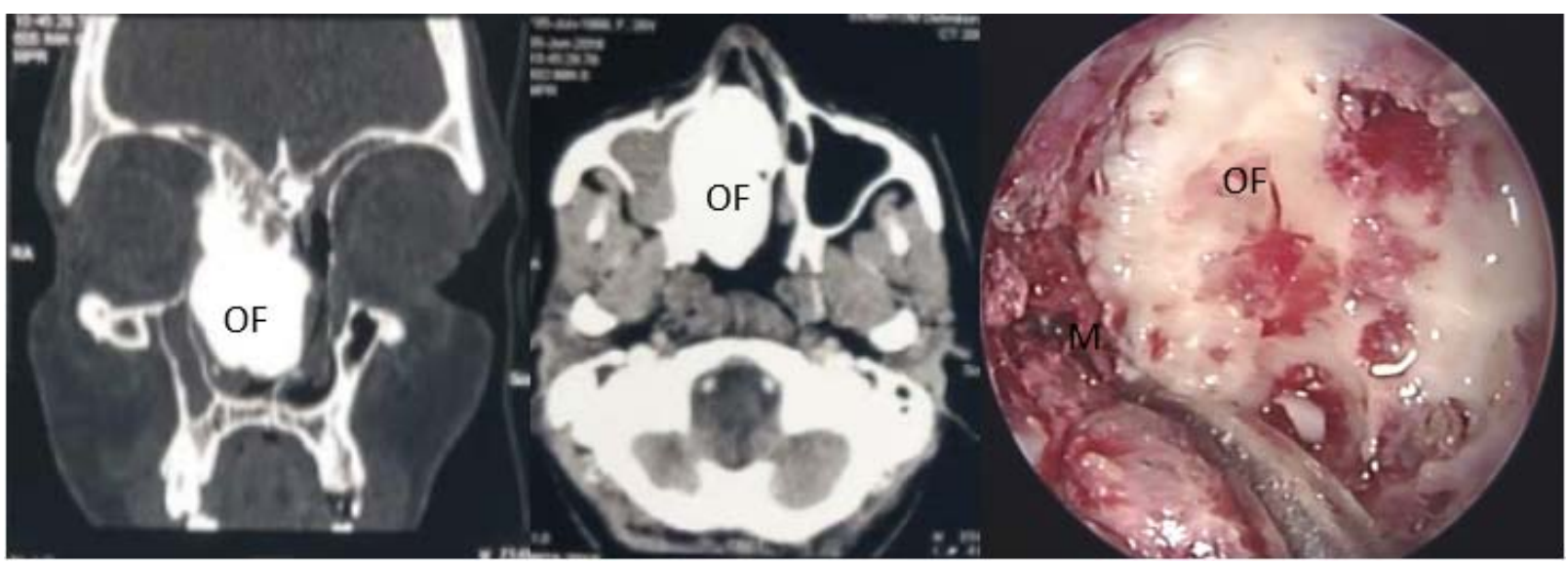

Figure 4. CT PNS axial and coronal image showing hyperdense image (OF) in right nasal cavity, ethmoidal region abutting right lamina cribrosa. Left sided intraoperative image shows opening of maxillary sinus along with tumour. M-opening of maxillary sinus, OF-ossifying fibroma. 


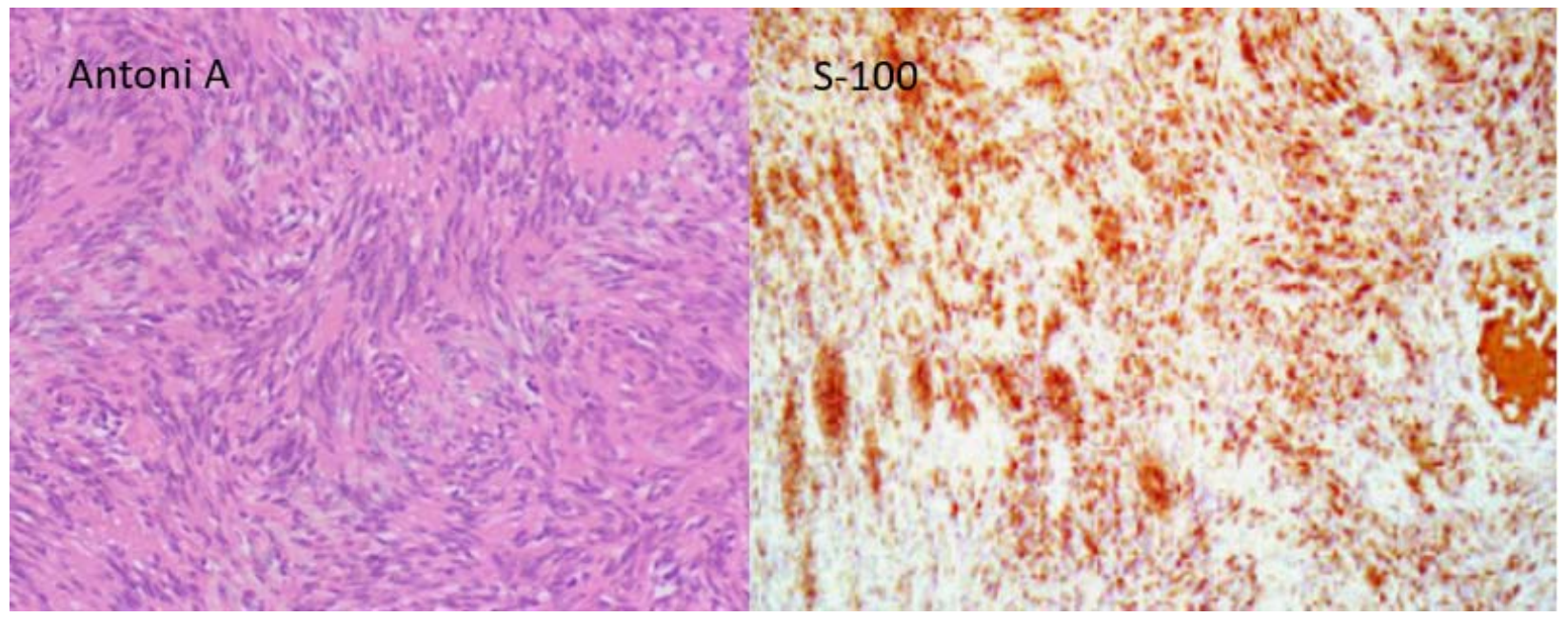

Figure 5. Right sided image shows HPE section of schwannoma showing Antoni-A pattern. Left sided image shows staining of schwannoma by S-100.

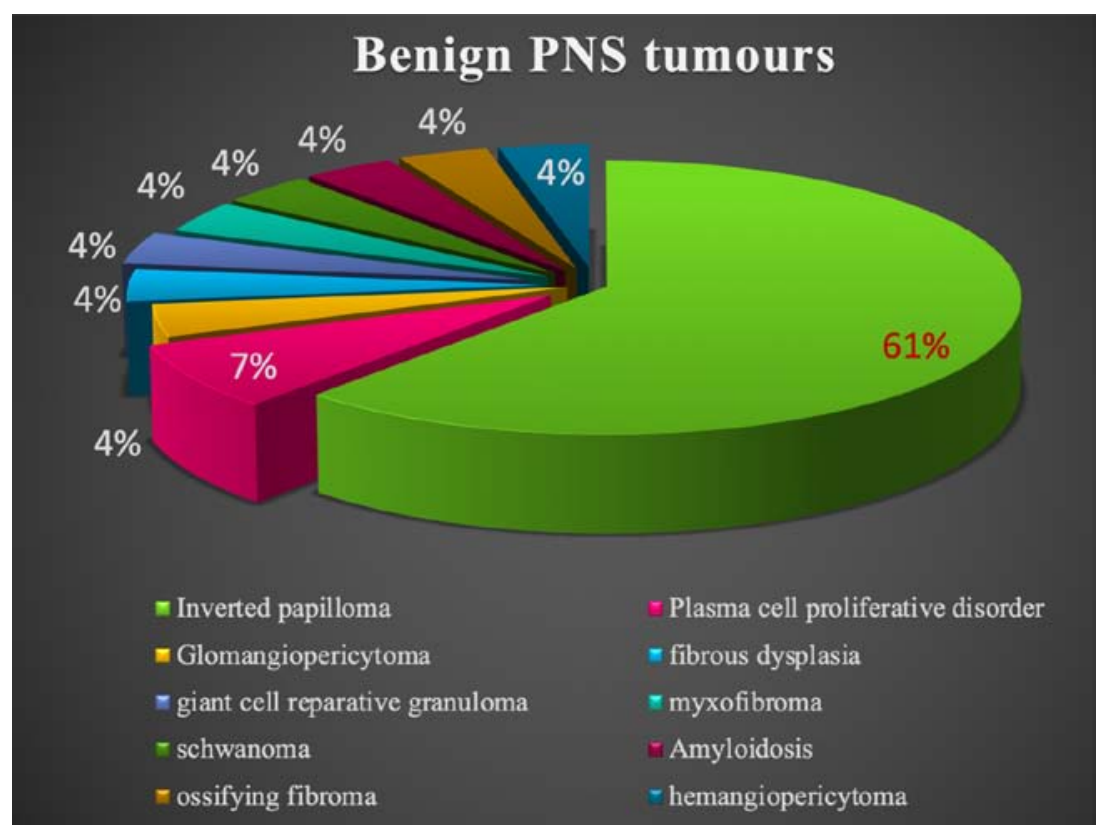

Figure 6. Distribution of benign PNS tumor.

\subsection{Plasmacytoma}

Plasma cell neoplasms (n-2, 7.7\%) were the second most common benign neoplasm of nasal cavity in our study. They are characterized by neoplastic proliferation of a single clone of plasma cells, producing a monoclonal immunoglobulin. They can present as a single lesion (solitary plasmacytoma) or as multiple lesions (multiple myelo$\mathrm{ma}$ ). Solitary plasmacytomas present as solitary plasmacytoma of bone (SPB), and extramedullary plasmacytoma (EMP) $[12,13]$. EMP are mainly located in the head and neck region, commonly affecting the nasal cavity, paranasal sinuses, tonsillar fossa, and oral cavity but may also occur in the gastrointestinal tract, urinary bladder, gland, lymph nodes, and skin [14, 35]. In nasal cavity, they represent approximately $4 \%$ of tumors, with median age of 55 to 60 years, with male predominance [14]. The etiology of this disease remains unknown, but chronic irritation from inhaled irritants and viral pathogenesis has been suggested as a cause [13]. Most patients present with symptoms such as epistaxis, nasal discharge (rhinorrhea) or nasal obstruction [14]. The main differential diagnosis is multiple myeloma and waldenstrom macroglobulinemia. Workup of a suspected EMP includes a biopsy of the suspected lesion for tissue histological confirmation, a unilateral bone marrow aspirate and biopsy, and laboratory studies. Imaging should include a metastatic bone survey and either a positron emission tomography/computed tomography (PET/CT) scan or magnetic resonance imaging (MRI) of the entire spine and pelvis [14, 15]. The treatment 
of choice for EMP is surgery and radiation therapy, the disease is highly radiosensitive. Small lesions may be cured with surgery alone and no adjuvant RT is indicated unless a residual local disease is suspicion [16]. In our study, 2 cases of paranasal plasmacytoma were operated endoscopically. Bone marrow of 1st patient showed no evidence of plasma cell proliferative disorder, Serum Kappa Free-light chain value was 58. 4mg/dl (3.3-19.4), Serum Lambda Free- light chain value was $29.3 \mathrm{mg} / \mathrm{dl}$ (5.71-26.3) with no M band on electrophoresis. Post operative radiotherapy was given to this patient as tumor was infiltrating planum sphenoidale. Bone marrow of second patient showed no evidence of plasma cell proliferative disorder, Serum Kappa Free-light chain value was 365 mg/dl (3.3-19.4), Serum Lambda Free- light chain value was $21.3 \mathrm{mg} / \mathrm{dl}$ (5.71-26.3) with M band on electrophoresis. Post operative radiotherapy was not given to this patient (Table 1). 5 year follow up of both patients showed no recurrence (Figure 7).

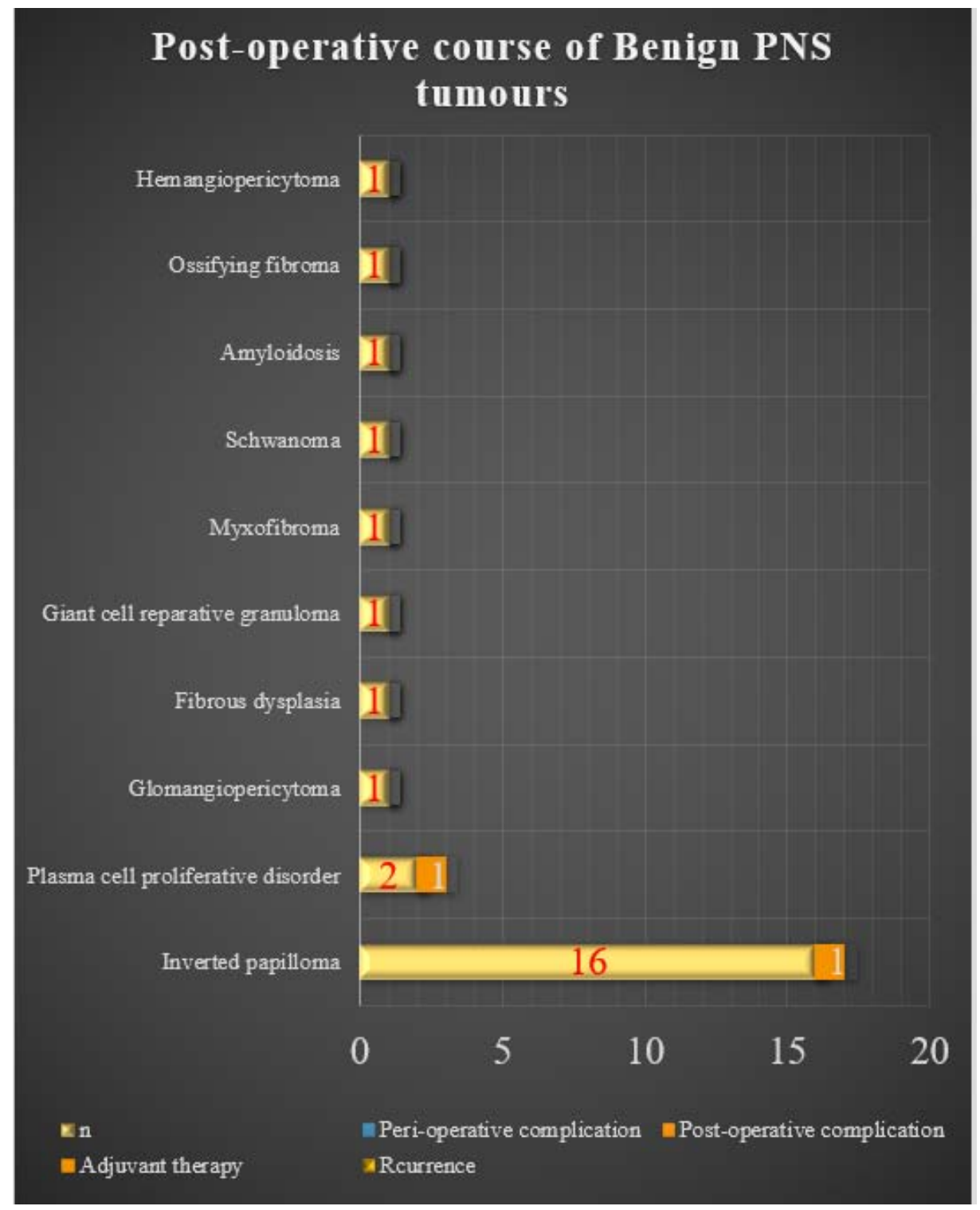

Figure 7. Post operative course of Benign PNS tumor/Peri-operative complications, Post-operative complications/Adjuvant therapy/Recurrence.

\subsection{Hemangiopericytoma (HPC)}

Hemangiopericytoma (n-1) (HPC) is a type of soft tissue sarcoma that originates in the pericytes in the walls of capillaries. Sino nasal hemangiopericytomas are extremely rare. Only few studies reported long-term results of surgical treatment of Sino nasal hemangiopericytoma [17, 18, 36]. Most of the authors consider these tumors clinically and biologically as benign lesions. Local recurrences were reported up to 13 years after surgical resection [17]. 
Metastasizing and locally aggressive HPC of head and neck was described in the literature [19]; however, in a study summarizing results of treatment of 104 Sino nasal hemangiopericytomas from different institutions for a 25-year period, there was no single case of a metastasizing tumor [20]. We had a 55-year female presenting with features of epistaxis, CECT revealed midline contrast enhancing mass. Patient underwent excision by transnasal endoscopic approach with no perioperative or postoperative complications (Figure 2). Perioperatively due to blood loss blood transfusion was done in post operative period. No recurrence or evidence of metastasis seen in 5 year follow up. (Table 1, Figure 7).

\subsection{Fibrous dysplasia}

Fibrous dysplasia (FD) (n-1) is a benign disease with slow progression. It is pathophysiologically characterized by the replacement of the normal bone by fibrous tissue. The bony trabeculae become irregularly placed, and a bone tissue with no internal lamellar structures develops [37]. It is associated with a gene mutation (especially GNAS1) [21]. Onset of disease is seen in childhood with progression during adolescence, while the incidence is decreased in the early adulthood [22]. FD accounts for $2.5 \%$ of bone tumors, whereas it accounts for $7.5 \%$ of the benign bone tumors [22]. The head and neck region is involved in $25 \%$ of the patients. In the head and neck region, the most frequently involved areas are the maxilla and mandible. Ethmoid bone is rarely involved. CT scan reveals features of ground glass appearance (Figure 3). T2-weighted MRI images in patients with FD reveal low signal from the involved bones, while ossifying fibromas give high signal intensity [23]. Lustig et al. reported in a series of 15 years that the ethmoid bone involvement was the most frequent one (71\%) among 21 patients in total. Definitive diagnosis of FD is made after a histopathological examination. In the histopathological examination, irregular trabeculae of the spongious bone as well as fibrous connective tissue, that is, stroma, are observed [23]. Various external approaches most frequently used to treat paranasal sinus fibrous dysplasia include the Caldwell luc technique, lateral rhinotomy, external ethmoidectomy, and craniofacial resection. Recently, more conservative trans-nasal endoscopic approaches have also been implemented [24]. In cases where the mass cannot be entirely excised, preferred approach is to debulk the lesion in the area where pressure is present [24]. Our study had an 18-year-old male with monostotic FD left ethmoid sinus, with clinical presentation of atypical pain and heaviness in the head and face along with sinusitis-related symptoms. On examination no proptosis, diplopia, and visual changes were observed. Transnasal endoscopic resection of the tumor was done with preservation of lamina papyrecea and skull base. No perioperative and post-operative complications were noticed. 5 year follow up showed no recurrence. (Table 1, Figure 7).

\subsection{Ossifying fibroma}

Ossifying fibroma (n-1, 3.8\%) involving the sinonasal tract is rare entity. Clinical presentations of this tumor are variable and depend upon the site and rate of growth of tumor [25]. Sino nasal tract involvement is generally asymptomatic and often diagnosed incidentally following radiographic examination. Symptomatic masses manifest by displacement of teeth or as an expansile mass [25]. Radiologic features of the lesion include the presence of a well-circumscribed or sharply demarcated lesion with smooth contours [25, 36]. Ossifying fibromas are composed of randomly distributed mature (lamellar) bone spicules rimmed by osteoblasts admixed with a fibrous stroma. While the osseous component is generally described as mature, the central portion may be woven bone with lamellar bone at the periphery. Complete bone maturation is seldom seen. The fibrous stroma may be densely cellular with absent mitotic activity [25].

In our study, we had 21-year female presented with right eye pain with proptosis, CT revealed a dense well circumscribed lesion involving right ethmoid sinus, lamina papyrecea along with skull base. Endoscopic excision of the tumor was done using a high-speed drill system, tumor involving the skull base was left for second stage surgery under navigation assistance to prevent any kind of intracranial injury (Figure 4, Table 1, Figure 7).

\subsection{Schwannoma}

Schwannoma/neurilemmoma (n-1, 3.8\%) is slow growing benign neoplasm arising from Schwann cells in the peripheral nerve sheath. Most common site of origin is being head and neck region [26]. Sinonasal schwannomas are rare, and represent less than $4 \%$ of the schwannomas of head and neck region , most common being ethmoidal sinus followed by maxillary sinus, nasal fossa and sphenoidal sinus [27]. Most common age group involved is between 0-78 years with no sex or racial predilection [27]. Chief complains are nasal obstruction, epistaxis, rhinorrhea, anosmia, facial swelling or pain [28]. Macroscopically schwannomas appear as gelatinous or cystic well encapsulated masses. Microscopically schwannomas are classified into two major histological types [29, 32]. Hypercellular 
or Antony A (Figure 5) areas comprised of spindle shaped cells arranged in interlacing fascicles and hypocellular or Antony B areas where the cells are present in a loose myxoid stroma. Parallel rows of palisading nuclei (verocay body) can be seen in highly differentiated tissue. The differential diagnosis includes inflammatory polyps, angiofibroma, inverted papilloma, meningiomas, neurofibroma, melanoma and neuroblastoma [29]. Histopathology remains gold standard for the diagnosis. Schwannomas usually show intense immunostaining for S-100 (Figure 5) which helps to distinguish peripheral nerve sheath tumor from others. The treatment is complete surgical excision which is determined according to the location and extent of lesion. Though recurrence is rare after removal, there are reports of malignant changes in long standing benign schwannoma [30]. Hence, long term and intimate follow up is required. We had one female presenting with complaints of nasal obstruction with headache, endoscopic biopsyrevealed schwannoma. Transnasal total excision was done with no perioperative and postoperative complication. No adjuvant therapy was given to patient. 5 year follow up of patient showed no recurrence (Table 1, Figure 7).

\section{Management protocol:}

\section{Preoperativemanagement:}

1. Signs and symptoms (epistasis, nasal obstruction, proptosis)

2. CECT PNS with MRI PNS with contrast

3. Diagnostic nasal examination with tissue biopsy (except JNA)

\section{Intraoperative management:}

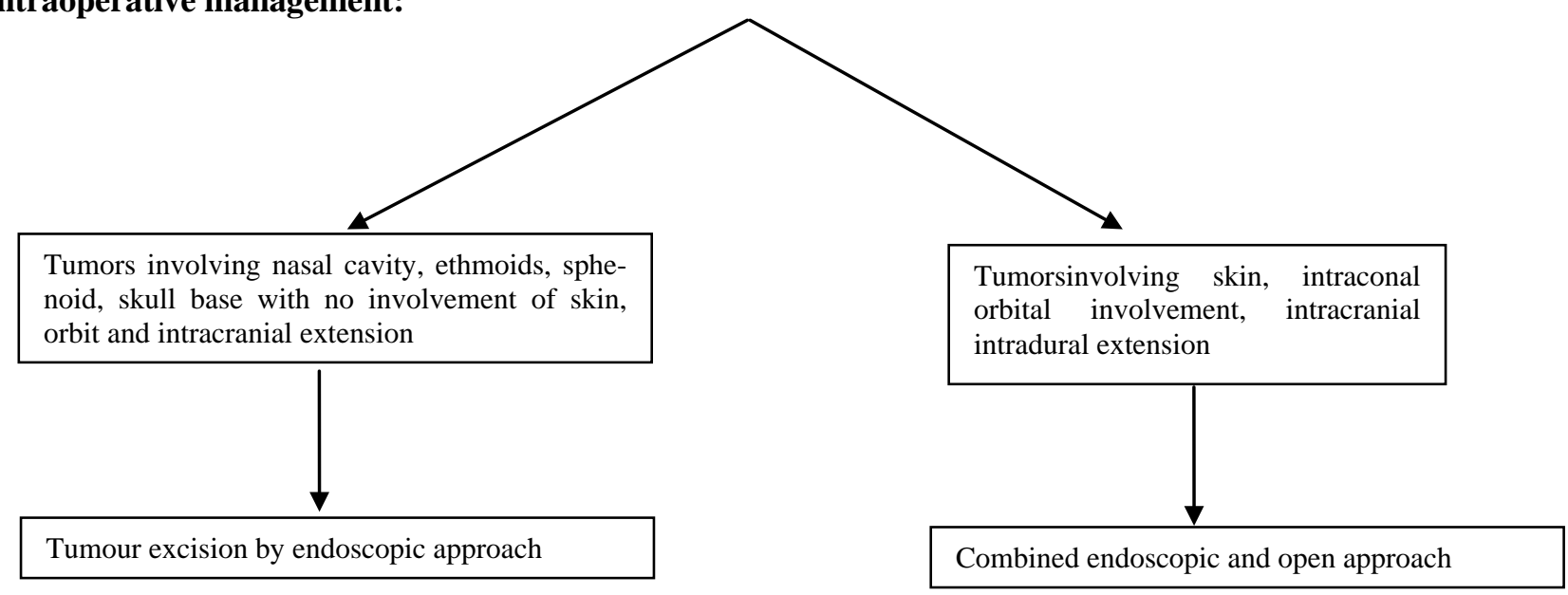

\section{Post operative management:}

1. Regular follow up is done 1 monthly with diagnostic nasal endoscopy for a period of 1 year for crust removal followed by 3 monthly for 2 years, followed by 6 monthlies till five years

2. CECT PNS is done 3 months following surgery to look for any recurrence

3. Patient is advised proper saline douching in post operative period

4. Adjuvant therapy (if needed, example: inverted papilloma carcinoma insitu, inverted papilloma converted into scc, plasmacytoma)

\section{Conclusions}

Endoscopic surgery of benign sinonasal tumor is as effective as external approach. Benign PNS and nasal cavity tumors not involving skin, orbit, tumors with intracranial extension can be managed by endoscopic excision followed by adjuvant therapy if needed (example: inverted papilloma carcinoma insitu, inverted papilloma converted into scc, plasmacytoma). A regular follow is needed for a better outcome.

\section{References}

[1] Thompson, L. D. R. (2006). Sinonasal carcinomas. Curr Diagn Pathol., 2006, 12: 40-53.

[2] Grau, C., Jakobsen, M. H., Harbo, G., et al. (2010). Sino-nasal cancer in Denmark 1982-1991—a nationwide survey. Acta Oncol., 2010, 40: 12-23.

[3] Lund, V., Stamberger, H., Nicolai, P., et al. (2010). European position paper on endoscopic management of tumors of the nose and paranasal sinuses and skull base. Rhinology, 2010, Suppl. 22: 1-143. 
[4] Carillo, J. F., Guemes, A., Ramirez-Ortega, M. C., Onate-Ocana, L. F. (2005). Prognostic factors in maxillary sinus and nasal cavity carcinoma. EJSO, 2005, 31: 1206-12.

[5] Żyłka, S., Bień, S., Kamiński, B., et al. (2008). Epidemiology and clinical characteristics of the sinonasal malignancies. Otolaryngol Pol., 2008, 62: 436-41.

[6] Ketcham, A. S., Wilkins, R. H., Vanburen, J. M., Smith, R. R. (1963). A combined intracranial facial approach to the paranasal sinuses. Am J Surg., 1963, 106: 698-703.

[7] Nicolai, P., Castelnuovo, P., Villaret, A. B. (2011). Endoscopic resection of sinonasal malignancies. Curr Oncol Rep., 2011, 13: 138-44.

[8] Nicolai, P., Battaglia, P., Bignami, M., et al. (2008). Endoscopic surgery for malignant tumors of the sinonasal tract and adjacent skullbase: a 10-year experience. Am J Rhinol., 2008, 22: 308-16.

[9] Hanna, E., DeMonte, F., Ibrhim, S., et al. Endoscopic resection of sinonasal cancers with and without craniotomy. Arch Otolaryngol Head Neck Surg., 2009, 135: 1219-24.

[10] Lombardi, D., Tomenzoli, D., Butta, L., et al. (2011). Limitations and complications of endoscopic surgery for treatment for sinonasal inverted papilloma: a re-assessment after 212 cases. Head Neck, 2011, 33: 1154-61.

[11] Krouse, J. H. (2000). Development of a staging system for inverted papilloma. Laryngoscope, 2000 Jun, 110(6): 965-8.

[12] Dores, G. M., Landgren, O., McGlynn, K. A., Curtis, R. E., Linet, M. S., Devesa, S. S. (2009). Plasmacytoma of bone, extramedullary plasmacytoma, and multiple myeloma: incidence and survival in the United States, 1992-2004. Br J Haematol., 2009, 144(1): 86-94.

[13] Soutar, R., Lucraft, H., Jackson, G., Reece, A., Bird, J., Low E., et al. (2004). Guidelines on the diagnosis and management of solitary plasmacytoma of bone and solitary extramedullary plasmacytoma.

[14] Bachar, G., Goldstein, D., Brown, D., Tsang, R., Lockwood, G., PerezOrdonez, B., et al. (2008). Solitary extramedullary plasmacytoma of the head and neck-long-term outcome analysis of 68 cases. Head Neck, 2008, 30(8): 1012-9.

[15] Creach, K. M., Foote, R. L., Neben-Wittich, M. A., Kyle, R. A. (2009). Radiotherapy for extramedullary plasmacytoma of the head and neck. Int J Radiat Oncol Biol Phys., 2009, 73(3): 789-94.

[16] Rajkumar, S. V., Dispenzieri, A., Kyle, R. A. (2006). Monoclonal gammopathy of undetermined significance, Waldenstrom macroglobulinemia, AL amyloidosis, and related plasma cell disorders: diagnosis and treatment. Mayo Clin Proc., 2006, 81(5): 693-703.

[17] Gomez-Rivera, F., Fakhri, S., Williams, M. W., et al. (2012). Surgical management of sinonasal hemangiopericytomas: a case series. Head Neck, 2012, 34: 1492-6.

[18] Bignami, M., Dallan, I., Battaglia, P., et al. (2010). Endoscopic, endonasal management of sinonasal hemangiopericytoma: 12-year experience. J Laryngol Otol., 2010, 124: 1178-82.

[19] Billings, K. R., Fu, S. Y., Calcaterra, T. C., Sercarz, J. A. (2000). Hemangiopericytoma of head and neck. Am J Otolaryngol., 2000, 21: 238-43.

[20] Thmpson, L. D. R., Miettinen, M., Wenig, B. M. (2003). Sinonasal-type hemangiopericytoma. A clinicopathological and immunophenotypic analysis of 104 cases showing perivascular and myoid differentiation. Am J Surg Pathol., 2003, 27: 737-49.

[21] Jia, S., Yu, J., Zhang, D., et al. (2011). Expression and regulation of amphiregulin in Gs a -mutated human bone marrow stromal cells of fibrous dysplasia of mandible. Oral Surg Oral Med Oral Pathol Oral Radiol Endod., 2011, 111: 618-626.

[22] Rojas, R., Palacios, E., Kaplan, J., et al. (2004). Fibrous dysplasia of the frontal sinus. Ear Nose Throat J., 2004; 83: 14-15.

[23] Engelbrecht, V., Preis, S., Hassler, W., et al. (1999). CT and MRI of congenital sinonasal ossifying fibroma. Neuroradiology 1999, 41: 526-529.

[24] Tsai, T. L., Ho, C. Y., Guo, Y. C., et al. (2003). Fibrous dysplasia of the ethmoid sinus. J Chin Med Assoc., 2003, 66: 131-133.

[25] Lam, S. Y., Ramli, N. M., Harikrishnan, D., Sia, S. F., Pailoor, J. (2008). Ossifying fibroma of the occipital bone-A case report and literature review. Eur J Radiolog Extra., 2008, 67(1): 19-23.

[26] Suh, J. D., Ramkrishnan, V. R., Zhang, P. J. (2011). Daignosis and endoscopic management of sinonasal schwannomas. ORL J Otorhino-Laryngol Relat Spec., 2011, 73: 308-12.

[27] Berlucchi, M., Piazza, C., Blanzuoli, L., Nicolai, P. (2000). Schwannoma of the nasal septum: a case report with review of literature. Eur Arch Otorhinolaryngol., 2000, 257: 402-05.

[28] Hegazy, H. M., Snydermann, C. H., Fan, C. Y., Kassam, A. B. (2001). Neurilemmomas of the paranasal sinuses. Am J Otolaryngol., 2001, 22: 215-18.

[29] Rodriguez, F. J., Folpe, A. L., Giannini, C., Perry, A. (2012). Pathology of peripheral nerve sheath tumours: diagnostic overview and update on selected diagnostic problems. Acta Neuropathol, 2012, 123: 295-319.

[30] Kurtkaya-Yapcer, Ö., Scheithauer, B., Woodruff, J. M. (2003). The pathobiologic spectrum of Schwannomas. HistolHistopathol., 2003, 18: 925-34. 
[31] V. J. Lund, P. M. Clarke, A. C. Swift, G. W. McGarry, C. Kerawala, and D. Carnell. (2016). Nose and paranasal sinus tumours: United Kingdom National Multidisciplinary Guidelines. J Laryngol Otol., 2016 May, 130(Suppl 2): S111-S118. doi: 10. 1017/S0022215116000530.

[32] Venkateswarlu, V., Janardhan, N., Chaitanya, V. K., et al. (2016). Schwannoma of the nasal septum: a case report. J. Evolution Med. Dent. Sci., 2016, 5(21): 1137-1139, DOI: 10. 14260/jemds/2016/264.

[33] Wu, V., Siu, J., Yip, J., et al. (2018). Endoscopic management of maxillary sinus inverted papilloma attachment sites to minimize disease recurrence. J of Otolaryngol-Head \& Neck Surg., 47, 24(2018). https://doi. org/10. 1186/s40463-018-0271-1.

[34] Sotaro Fukuharaa, Hirofumi Tazawaa, Hideharu Okanobub, Michiko Kidac, Miki Kidoc, Toshiro Takafutac, Toshihiro Nishidad, Hideki Ohdane, Hideto Sakimotoae. (2016). Successful treatment of primary advanced gastric plasmacytoma using a combination of surgical resection and chemotherapy with bortezomib: A case report. International Journal of Surgery Case Reports, Volume 27, 2016, pp. 133-136.

[35] Zada, G., Lopes, M. B. S., Mukundan, S., Laws, E. (2016). Hemangiopericytoma. In: Zada G., Lopes M., Mukundan Jr. S., Laws Jr. E. (eds), Atlas of Sellar and Parasellar Lesions. Springer, Cham. https://doi. org/10. 1007/978-3-319-22855-6_29.

[36] Javaid, M. K., Boyce, A., Appelman-Dijkstra, N., et al. (2019). Best practice management guidelines for fibrous dysplasia/McCune-Albright syndrome: a consensus statement from the FD/MAS international consortium. Orphanet J Rare Dis., 14, 139(2019). https://doi. org/10. 1186/s13023-019-1102-9.

[37] Shin Saitoa, Hiroyuki Ozawaa, YuuichiI karia, Nana Nakaharaa, Fumihiro Itob, Mariko Sekimizua, Kaori Kameyamac, Takenori Akiyamad, Masahiro Todad, Kaoru Ogawaa. (2020). Endoscopic endonasal removal of a pediatric paranasal ossifying fibroma using preoperative embolization. Otolaryngology Case Reports Volume 14, March 2020, 100147. 